\title{
Simulating the phase fluctuations produced by many stars in the weak atmospheric turbulence limit
}

\author{
V. V. Voitsekhovich ${ }^{1}$, D. Kouznetsov ${ }^{2}$, and V. G. Orlov ${ }^{1}$ \\ ${ }^{1}$ Instituto de Astronomia, UNAM. AP 70-264 Cd. Universitaria, 04510 Mexico D.F., Mexico \\ e-mail: voisteko@astroscu.unam.mx \\ 2 Mathematics Building, University of Arizona, USA
}

Received 3 January 2005 / Accepted 21 June 2005

\section{ABSTRACT}

We propose a method allowing for a simulation of cross-correlated turbulence-induced phase fluctuations produced by many stars is proposed. It turns out that for weak-turbulence conditions which are important for many applications, it is possible to suggest a relatively simple and fast simulation procedure. Both the variation of $C_{n}^{2}$ along a propagation path and an effect of finite outer scale of the turbulence are considered. The validity of the simulation is verified by comparing theoretical and simulated results, and it is shown that there is good agreement takes place even for big separations between the observation points. Adaptive optics, guide stars, speckle interferometry, long-base interferometry, imaging through the turbulence, as well as simulations of the image distortions of extended objects are among the possible applications of this method.

Key words. instumentation: adaptive optics - atmospheric effects - methods: numerical - telescopes

\section{Introduction}

Simulation of turbulence-induced cross-correlated phase fluctuations to be produced by many stars on the observation plane is important for a wide range of applications. Among them are adaptive optics (Shapiro 1976; Fried 1982; Fusco et al. 2001; Berkefeld et al. 2001), guide stars (Foy \& Laberye 1985; Tallon \& Foy 1990; Schock et al. 2002; Fried 1995), speckle interferometry (Roddier 1981; Dainty 1974; Roddier et al. 1982), imaging through the turbulence (Korff 2001; Ricklin et al.1995; Vorontsov et al. 1996), imaging of extended objects, etc. However, in many cases of practical concern, it is extremely difficult (or even impossible) to treat the problems of interest with purely theoretical methods, while the corresponding simulations can provide the needed solutions for both physical and technical problems related to the above areas.

A simple example of such a problem is calculation of statistical characteristics of turbulence-degraded image produced by many stars at the telescope focal plane. While it is quite complicate to resolve this problem theoretically, one can obtain interesting results by making use of simulations in a relatively simple and straight forward way. Using simulations, the calculation procedure can be as follows: first to generate phase fluctuations samples at the aperture, then to compute the images corresponding to each sample, and finally to calculate the statistical quantities of interest in these images. Also, the simulations have another advantage since by observing separated samples one can sence the physics of a process under investigation better. The main problem in the case of such simulations is generating the phase samples with the proper statistics and cross-statistics at the aperture, so in this paper we present such a method.

Analyzing the simulation approaches used in turbulencerelated problems, one can distinguish two main categories of methods. In the first one (Martin \& Flatte 1988), the extended random media are divided into slabs (phase screens) in the propagation direction assuming that each of them gives an independent contribution to the wave phase. The spectrum of refractive-index fluctuations associated with each screen is assumed to be known from theory, and the corresponding refractive-index samples are usually generated by filtering white Gaussian noise. The samples of wave field on the observation plane are found either by considering the geometric optics propagation between the screens, Fresnel propagation, or through a direct numerical solution of the parabolic or wave equation.

In the second category of methods, the statistics of simulated process on the observation plane is assumed to be known from theory. The process to be simulated is expanded over a set of some basic functions (Fourier series: Sedmak 2004; Louthain \& Welsh 1998, Zernike polynomials: Roddier 1990, Karhunen-Loeve functions: Cannon 1995; Orlov et al. 1998, etc.), and the expansion coefficients are sought in such a way as to allow one to reproduce the given statistics in simulated samples. 
The method of random wave vectors (RWV) (Kouznetsov et al. 1997; Kouznetsov \& Voitsekhovich 1998), which we apply for the simulation, belongs to the second category. As in conventional approaches, the RWV method suggests construction of the simulated process through its expansion in the domain of spatial frequencies. On first glance, the RWV expansion looks like the usual two-dimensional Fourier series. However, it is the only similarity in the two approaches, and one main difference between the expansions is in the choice of spatial frequencies. In contrast to the Fourier method, where the dynamic range is determined by the grid parameters, the RWV method suggests a more flexible approach that allows us to significantly widen the dynamic range of simulation. We discuss this remarkable feature of the RWV method in more detail in the last section of this paper. The computational efficiency of the RWV method is compared to the Fourier approach in Kouznetsov \& Voitsekhovich (1998), while comparison to some other simulation techniques is presented in Kouznetsov et al. (1997).

In this paper we apply the RWV method for simulation of cross-correlated phase fluctuations due to the propagation of many initially plane waves through the weak turbulence. As will be seen, a simulation procedure is relatively simple because the cross-spectra of phase fluctuations produced by the weak turbulence have certain specific properties.

The paper is organized as follows. In Sect. 2 we present the theoretical expressions for the correlation and cross-correlation functions, spectra, and cross-spectra of the turbulence-induced phase fluctuations to be produced by off-axis stars. All these quantities are calculated in the Rytov approximation (Tatarski 1961). In Sect. 3 we derive all the formulas needed for the simulation. A possibility of simultaneously handling all the cross-correlations in such a relatively simple way is due to some specific properties of the weak-turbulence-induced phase fluctuations. At the end of Sect. 3 we also give a detailed recipe of the simulation procedure. Section 4 presents the simulation results and compares them to the theoretical ones, while Sect. 5 concludes the paper. In that section a short summary of the results is given and the main advantages of the method are discussed.

\section{Theoretical correlations and cross-correlations}

In order to apply the RWV method to simulation of crosscorrelated phase fluctuations produced by many stars, one needs to know the spectra and the cross-spectra of these fluctuations on the observation plane. In this paper we restrict our attention to the simulation of cross-correlated phase fluctuations due to the propagation of monochromatic light waves through the weak turbulence. Under weak-turbulence conditions the necessary spectra and cross-spectra follow from the approximated analytical Rytov solution of the parabolic equation (Tatarski 1961). The Rytov method is actually the conventional perturbation method but applied to the logarithm of the field rather than to the field itself. As a result the Rytov method gives a solution for the logarithm of the field, which is also called a complex amplitude. Compared to the usual Born approximation, which gives a solution for the field, the Rytov one has the advantage of partially handling the effect of multiple scattering (Tatarski 1961).

In this section, we first calculate the correlation and crosscorrelation functions of phase fluctuations on the observation plane using the results of the Rytov solution. Then, applying the Fourier transform to these functions, we get the corresponding spectra and cross-spectra needed for the simulation.

Let us consider $L$ stars and assume that these stars produce $L$ monochromatic, initially plane waves at the upper boundary of the atmosphere. Then, let us introduce the threedimensional Cartesian coordinate system with the $\mathrm{X}$ and $\mathrm{Y}$ axes placed on the aperture plane. Furthermore, let the $l$ th wave have the propagation unit vector $\xi_{l}$ at the upper boundary of the atmosphere. The Rytov solution for the fluctuation $\Psi_{l}$ of the complex amplitude to be produced by the $l$ th star at the observation plane is expressed as (Voitsekhovich et al. 1998):

$$
\begin{aligned}
& \begin{aligned}
\Psi_{l}(\boldsymbol{r}) & =\chi_{l}(\boldsymbol{r})+\mathrm{i} S_{l}(\boldsymbol{r}) \\
& =\mathrm{i} k \int_{0}^{H} \mathrm{~d} z \int \mathrm{d}^{2} \kappa g_{n}(z, \boldsymbol{\kappa}) \exp \left(\mathrm{i} \boldsymbol{r} \cdot \boldsymbol{r}+\frac{\kappa^{2} z}{2 \mathrm{i} k}-\mathrm{i} \boldsymbol{\kappa} \cdot \boldsymbol{n}_{l} z\right),
\end{aligned} \\
& \boldsymbol{n}_{l}=\left(\sin \gamma_{l} \cos \alpha_{l}, \sin \gamma_{l} \sin \alpha_{l}\right),
\end{aligned}
$$

where $\chi_{l}$ and $S_{l}$ denote the log-amplitude and the phase fluctuation produced by the $l$ th star, respectively; $\boldsymbol{r}=(x, y)$ denotes the two-dimensional (2-D) position vector at the observation plane; $k$ is the wavenumber, $H$ denotes the propagation distance, $g_{n}$ the spectrum of fluctuations of the refractive-index random field, $\boldsymbol{n}_{l}$ is the projection of the vector $\boldsymbol{\xi}_{l}$ on the $X Y$ plane, $\gamma_{l}$ the angle between the $l$ th star and $Z$-axis, and $\alpha_{l}$ the polar angle of the vector $\boldsymbol{n}_{l}$. More detailed explanations related to the quantity $g_{n}$ can be found in Tatarski (1961).

The way to calculate the correlation function $B_{S}$ of phase through the complex amplitude fluctuation $\Psi$ is described in Tatarski (1961), so we present here only the main formulas. As it follows from the definition (1) of the $\Psi$, the correlation function $B_{S}$ of the phase $S$ is expressed as $\boldsymbol{s}$

$$
\begin{aligned}
B_{S}\left(\boldsymbol{r}_{1}, r_{2}\right) & =\left\langle S\left(\boldsymbol{r}_{1}\right) S\left(\boldsymbol{r}_{2}\right)\right\rangle \\
& =\frac{1}{2} \operatorname{Im}\left[\left\langle\Psi\left(\boldsymbol{r}_{1}\right) \Psi^{*}\left(\boldsymbol{r}_{2}\right)\right\rangle-\left\langle\Psi\left(\boldsymbol{r}_{1}\right) \Psi\left(\boldsymbol{r}_{2}\right)\right\rangle\right],
\end{aligned}
$$

where $\langle\cdots\rangle$ denotes the statistical average, Im denotes the imaginary part of complex quantity, and the asterisk $(*)$ denotes the complex conjugation.

The procedure for averaging the quantities $g_{n}$ taking place in Eq. (1) is performed with the help of the following equalities (Tatarski 1961)

$$
\begin{aligned}
& \left\langle g_{n}\left(z_{1}, \boldsymbol{\kappa}_{1}\right) g_{n}^{*}\left(z_{2}, \boldsymbol{\kappa}_{2}\right)\right\rangle=2 \pi \Phi_{n}\left(z_{1}, \boldsymbol{\kappa}_{1}\right) \delta\left(z_{1}-z_{2}\right) \delta\left(\boldsymbol{\kappa}_{1}-\boldsymbol{\kappa}_{2}\right), \\
& \left\langle g_{n}\left(z_{1}, \boldsymbol{\kappa}_{1}\right) g_{n}\left(z_{2}, \boldsymbol{\kappa}_{2}\right)\right\rangle=2 \pi \Phi_{n}\left(z_{1}, \boldsymbol{\kappa}_{1}\right) \delta\left(z_{1}-z_{2}\right) \delta\left(\boldsymbol{\kappa}_{1}+\boldsymbol{\kappa}_{2}\right),
\end{aligned}
$$

where $\Phi_{n}$ denotes the spectrum of refractive-index fluctuations, and $\delta$ is the Dirac delta-function. 
Using Eqs. (2) and (3), we can calculate from Eq. (1) the correlation functions $B_{S_{I} S_{l}}$ and the cross-correlation functions $B_{S_{l} S_{j}}$ of phase fluctuations as follows

$$
\begin{aligned}
B_{S_{l} S_{l}}(\rho) & =4 \pi^{2} k^{2} \int_{0}^{H} \mathrm{~d} z \int \kappa \kappa \Phi_{n}(z, \kappa) J_{0}(\kappa \rho), \\
l=1, \ldots, L & \\
B_{S_{l} S_{j}}(\boldsymbol{\rho}) & =\left\langle S_{l}\left(\boldsymbol{r}_{1}\right) S_{j}\left(\boldsymbol{r}_{2}\right)\right\rangle \\
& =4 \pi^{2} k^{2} \int_{0}^{H} \mathrm{~d} z \int \mathrm{d} \kappa \kappa \Phi_{n}(z, \kappa) J_{0}\left(\kappa\left|\boldsymbol{\rho}-\left(\boldsymbol{n}_{l}-\boldsymbol{n}_{j}\right) z\right|\right),
\end{aligned}
$$

$l=1, \ldots, L ; j=1, \ldots, L$

where $\boldsymbol{\rho}=\boldsymbol{r}_{1}-\boldsymbol{r}_{2}, \rho$ and $\kappa$ denote the modules of the corresponding vectors, $J_{0}$ is the Bessel function, and $|\cdots|$ stands for the modulus of the vector. In derivation of Eqs. (4), (5), the following usual assumptions have been used (Tatarski 1961): (i) the isotropy of the refractive-index spectrum $\Phi_{n}$; (ii) the approximate equality $1+\cos \left(\frac{\kappa^{2} z}{k}\right) \approx 2$ that holds with good accuracy in calculations of the statistics of turbulence-induced phase fluctuations.

In what follows we use the von Karmann refractive-index spectrum $\Phi_{n}$ (Reinhardt \& Collins 1972) to include the outer scale effects:

$$
\Phi_{n}(z, \kappa)=0.033 C_{n}^{2}(z)\left(\kappa^{2}+1 / L_{0}^{2}\right)^{-11 / 6},
$$

where $C_{n}^{2}(z)$ is the profile of the refractive-index structure characteristic, and $L_{0}$ denotes the outer scale of the turbulence.

Substituting Eqs. (6) into (5) we have

$$
\begin{aligned}
& B_{S_{l} S_{l}}(\rho)= \\
& \quad 0.132 \pi^{2} k^{2} \int_{0}^{H} \mathrm{~d} z C_{n}^{2}(z) \int_{0}^{\infty} \mathrm{d} \kappa \kappa\left(\kappa^{2}+\frac{1}{L_{0}^{2}}\right)^{-11 / 6} J_{0}(\kappa \rho),
\end{aligned}
$$$$
l=1, \ldots, L,
$$

$$
\begin{aligned}
B_{S_{l} S_{j}}(\boldsymbol{\rho})= & 0.132 \pi^{2} k^{2} \int_{0}^{H} \mathrm{~d} z C_{n}^{2}(z) \int_{0}^{\infty} \mathrm{d} \kappa \kappa\left(\kappa^{2}+1 / L_{0}^{2}\right)^{-11 / 6} \\
& \times J_{0}\left(\kappa\left|\boldsymbol{\rho}-\left(\boldsymbol{n}_{l}-\boldsymbol{n}_{j}\right) z\right|\right)
\end{aligned}
$$

$l=1, \ldots, L ; j=1, \ldots, L$.

Introducing the two-dimensional spectrum $W(\boldsymbol{\kappa})$ of the function $B(\rho)$ as

$W(\boldsymbol{\kappa})=\frac{1}{2 \pi} \int \mathrm{d}^{2} \rho B(\boldsymbol{\rho}) \exp (\mathrm{i} \boldsymbol{\kappa} \cdot \boldsymbol{\rho})$,

and applying the transformation (9) to Eqs. (5), (6), we get the following expressions for the spectra $W_{S_{l} S_{l}}$ and for the crossspectra $W_{S_{l} S_{j}}$ of phase fluctuations

$W_{S_{l} S_{l}}(\kappa)=0.132 \pi^{2} k^{2}\left(\kappa^{2}+1 / L_{0}^{2}\right)^{-11 / 6} \int_{0}^{H} \mathrm{~d} z C_{n}^{2}(z)$,

$l=1, \ldots, L$,

$$
\begin{aligned}
& \operatorname{Re} W_{S_{l} S_{j}}(\kappa)=0.132 \pi^{2} k^{2}\left(\kappa^{2}+1 / L_{0}^{2}\right)^{-11 / 6} \\
& \times \int_{0}^{H} \mathrm{~d} z C_{n}^{2}(z) \cos \left[\boldsymbol{\kappa} \cdot\left(\boldsymbol{n}_{l}-\boldsymbol{n}_{j}\right) z\right], \\
& \operatorname{Im} W_{S_{l} S_{j}}(\boldsymbol{\kappa})=0.132 \pi^{2} k^{2}\left(\kappa^{2}+1 / L_{0}^{2}\right)^{-11 / 6} \\
& \times \int_{0}^{H} \mathrm{~d} z C_{n}^{2}(z) \sin \left[\boldsymbol{\kappa} \cdot\left(\boldsymbol{n}_{l}-\boldsymbol{n}_{j}\right) z\right], \\
& l=1, \ldots, L ; j=1, \ldots, L,
\end{aligned}
$$

where $\operatorname{Re}$ and Im denote the real and the imaginary parts of complex quantity.

\section{Simulation method: theoretical derivations}

We want to simulate the phase fluctuations $S_{l}(\boldsymbol{r})$ in such a way that all the simulated correlation functions $B_{S_{l} S_{l}}(l=1, \ldots, L)$ and the cross-correlation functions $B_{S_{l} S_{j}}$ $(l=1, \ldots, L ; j=1, \ldots, L)$ coincide with the corresponding theoretical ones given by Eqs. (5), (6). Because the phase fluctuations produced by the weak turbulence are Gaussian (Tatarski 1961), these coincidence conditions are sufficient to reproduce the statistics and cross-statistics of any order in simulated samples. As will be seen in what follows, the conditions of coincidence are satisfied if the phase fluctuations $S_{l}$ at the observation plane are simulated as

$S_{l}(\boldsymbol{r})=\sum_{m=1}^{M} F\left(p_{m}\right) \cos \left(\boldsymbol{p}_{m} \cdot \boldsymbol{r}+\varphi_{m}-\boldsymbol{p}_{m} \cdot \boldsymbol{n}_{l} \psi_{m}\right)$,

$l=1, \ldots, L$

where $\boldsymbol{r}$ is the 2-D position vector at the aperture plane, $M$ denotes the number of harmonics used in simulation, $\boldsymbol{p}_{m}$ is the 2 -D random wave vector, $p_{m}$ the modulus of the vector $\boldsymbol{p}_{m}$, and the vector $\boldsymbol{n}_{l}$ is given in Eq. (1). The meaning of the rest of parameters is explained in what follows.

The following statistical restrictions are imposed on the parameters in Eq. (12):

(j) the modules $p_{m}$ and the orientations $\theta_{m}$ of the vectors $\boldsymbol{p}_{m}$, the quantities $\varphi_{m}$ and $\psi_{m}$ are considered as statistically independent quantities;

(jj) $\theta_{m}$ and $\varphi_{m}$ are distributed uniformly inside the range $[-\pi, \pi]$.

With the conditions (j) and (jj) in hand, Eq. (12) allows for a direct physical interpretation. The statistical independence of $\varphi_{m}$ and their uniform distribution inside the range $[-\pi, \pi]$ restrict consideration to the homogeneous processes. With these restrictions, only the processes with the correlation function $B\left(\boldsymbol{r}_{1}, \boldsymbol{r}_{2}\right)=B\left(\boldsymbol{r}_{1}-\boldsymbol{r}_{2}\right)$ can be simulated. Then, only the isotropic processes are chosen from the class of homogenous ones thereby imposing the random uniform distribution inside the range $[-\pi, \pi]$ for the orientations $\theta_{m}$ of the vectors $\boldsymbol{p}_{m}$. In other words, with these two restrictions we allow only the isotropic processes with $B\left(\boldsymbol{r}_{1}, \boldsymbol{r}_{2}\right)=B\left(\left|\boldsymbol{r}_{1}-\boldsymbol{r}_{2}\right|\right)$ to be simulated. The isotropy of simulated processes also permits choosing the functions $F\left(p_{m}\right)$ independent of the orientation of the 
vector $\boldsymbol{p}_{m}$. Furthermore, the term $\boldsymbol{p}_{m} \cdot \boldsymbol{n}_{l} \psi_{m}$ reproduces the needed cross-correlations among the processes.

Now we can continue our consideration seeking the magnitudes $F\left(p_{m}\right)$ and proper statistical distributions for $\boldsymbol{p}_{m}$ and $\psi_{m}$. We choose these quantities in such a way that the correlation and cross-correlation functions simulated with Eq. (12) coincide with the corresponding theoretical ones (5), (6).

Using the same development as in Ref. (23), we can write a condition of coincidence for the correlation and crosscorrelation functions as

$$
\begin{aligned}
& B_{S_{l} S_{l}}(\rho)=\frac{1}{2} \sum_{m=1}^{M}\left\langle F^{2}\left(p_{m}\right) \cos \left(\boldsymbol{p}_{m} \cdot \boldsymbol{\rho}\right)\right\rangle, l=1, \ldots, L \\
& B_{S_{l} S_{j}}(\boldsymbol{\rho})=\frac{1}{2} \sum_{m=1}^{M}\left\langle F^{2}\left(p_{m}\right) \cos \left[\boldsymbol{p}_{m} \cdot \boldsymbol{\rho}-\boldsymbol{p}_{m} \cdot\left(\boldsymbol{n}_{l}-\boldsymbol{n}_{j}\right) \psi_{m}\right]\right\rangle \\
& l=1, \ldots, L ; j=1, \ldots, L
\end{aligned}
$$

Now we need to perform the statistical average in Eqs. (11), (12). In other words, the short-hand notation $\langle\cdots\rangle$ has to be replaced by the corresponding probability density functions (PDF). Such a procedure is easily performed in the Fourier domain. Thus, applying the two-dimensional Fourier transform (9) to Eqs. (11), (12), we can rewrite the condition of the coincidence in terms of the spectra and cross-spectra as

$$
\begin{aligned}
W_{S_{l} S_{l}}(\kappa)= & \frac{\pi}{2} \sum_{m=1}^{M} F \int \mathrm{d}^{2} p_{m} F^{2}\left(p_{m}\right) \Omega\left(\boldsymbol{p}_{m}\right) \\
& \times\left[\delta\left(\boldsymbol{p}_{m}+\boldsymbol{\kappa}\right)+\delta\left(\boldsymbol{p}_{m}-\boldsymbol{\kappa}\right)\right] \\
l=1, \ldots, L, & \\
W_{S_{l} S_{j}}(\boldsymbol{\kappa})= & \frac{\pi}{2} \sum_{m=1}^{M} F \int \mathrm{d} \psi_{m} \eta\left(\psi_{m}\right) \int \mathrm{d}^{2} p_{m} F^{2}\left(p_{m}\right) \Omega\left(\boldsymbol{p}_{m}\right) \\
& \times\left\{\exp \left[-\mathrm{i} \boldsymbol{p}_{m} \cdot\left(\boldsymbol{n}_{l}-\boldsymbol{n}_{j}\right) \psi_{m}\right] \delta\left(\boldsymbol{p}_{m}+\boldsymbol{\kappa}\right)\right. \\
& \left.+\exp \left[\mathrm{i} \boldsymbol{p}_{m} \cdot\left(\boldsymbol{n}_{l}-\boldsymbol{n}_{j}\right) \psi_{m}\right] \delta\left(\boldsymbol{p}_{m}-\boldsymbol{\kappa}\right)\right\}, \\
l=1, \ldots, L ; & j=1, \ldots, L,
\end{aligned}
$$

where $W_{S_{l} S_{l}}$ and $W_{S_{l} S_{j}}$ are given by Eqs. (10) and (11), respectively, and $\Omega$ and $\eta$ are the PDF's of the vector $\boldsymbol{p}_{m}$ and $\psi_{m}$, respectively.

Using Eq. (15), we can express the quantities $F(\kappa)$ in terms of the theoretical spectra and the PDF $\Omega$ as follows. Since the processes under simulation are isotropic, the PDF $\Omega(\kappa)$ of the vector $\boldsymbol{\kappa}$ can also be chosen as an isotropic function; i.e. $\Omega(\boldsymbol{\kappa})$ does not depend on the polar angle of the vector $\kappa$. Taking the isotropy of $\Omega(\kappa)$ into account and performing the integration over $p_{m}$ and the summation over $m$ in Eq. (15), we get $F(\kappa)$ as

$$
F(\kappa)=\sqrt{\frac{W_{S_{l} S_{l}}(\kappa)}{\pi M \Omega(\kappa)}}, l=1, \ldots, L .
$$

Note that there is a misprint in Ref. (21): the coefficient $\pi$ in Eqs. (6), (9)-(11) has to be replaced by $\pi / 2$.

Let us now choose a proper PDF $\eta\left(\psi_{m}\right)$ for $\psi_{m}$. We want to find such a PDF as would allow us to reproduce all the needed cross-correlations in simulated processes. Performing the integration over $p_{m}$ and the summation over $m$ in Eq. (16), and taking Eqs. (8), (9), (15) into account, one can get the following relationship between the PDF $\eta\left(\psi_{m}\right)$ and $C_{n}^{2}$ profile

$$
\begin{aligned}
& \int \mathrm{d} \psi_{m} \eta\left(\psi_{m}\right) \cos \left[\boldsymbol{\kappa} \cdot\left(\boldsymbol{n}_{l}-\boldsymbol{n}_{j}\right) \psi_{m}\right]= \\
& \quad \int_{0}^{H} \mathrm{~d} z C_{n}^{2}(z) \cos \left[\boldsymbol{\kappa} \cdot\left(\boldsymbol{n}_{l}-\boldsymbol{n}_{j}\right) z\right] / \int_{0}^{H} \mathrm{~d} z C_{n}^{2}(z), \\
& \int \mathrm{d} \psi_{m} \eta\left(\psi_{m}\right) \sin \left[\boldsymbol{\kappa} \cdot\left(\boldsymbol{n}_{l}-\boldsymbol{n}_{j}\right) \psi_{m}\right]= \\
& \int_{0}^{H} \mathrm{~d} z C_{n}^{2}(z) \sin \left[\boldsymbol{\kappa} \cdot\left(\boldsymbol{n}_{l}-\boldsymbol{n}_{j}\right) z\right] / \int_{0}^{H} \mathrm{~d} z C_{n}^{2}(z), \\
& l=1, \ldots, L ; j=1, \ldots, L .
\end{aligned}
$$

Since $C_{n}^{2}(z)$ is a non-negative function, we can always choose the PDF of $\psi_{m}$ in such a way that Eq. (18) will be satisfied for all the $l$ and $j$. The needed PDF $\eta\left(\psi_{m}\right)$ is given by

$\eta\left(\psi_{m}\right)=\left\{\begin{array}{l}C_{n}^{2}\left(\psi_{m}\right) / \int_{0}^{H} \mathrm{~d} z C_{n}^{2}(z), 0 \leq \psi_{m} \leq H \\ 0, \quad \text { otherwise. }\end{array}\right\}$

As one can see, the PDF (19) forces both Eqs. (18) to be the identities. From a practical point of view it means that if $F(\kappa)$ and $\eta\left(\psi_{m}\right)$ are determined from Eqs. (15), (17), respectively, simulation with Eq. (12) provides all the necessary correlations and cross-correlations among the simulated phase fluctuations. The PDF (19) can be generated for an arbitrary $C_{n}^{2}$ profile using, for example, the rejection method (Macakill \& Beran 1984).

Now we need to choose the PDF $\Omega(\boldsymbol{\kappa})$ of the vector $\boldsymbol{\kappa}$. From a mathematical point of view, $\Omega(\boldsymbol{\kappa})$ can be constructed in many different ways. However, we can restrict the choice using the following physical considerations. If we need to simulate a single process, the best choice would be to take $\Omega(\kappa)$ proportional to the square root of the spectrum of simulated process. Such an approach is in some sense similar to the well-known method for filtering out white Gaussian noise (Press et al. 1995). However, if we need to simulated several processes with different spectra, some compromise has to be found. In (Kouznetsov et al.1997), the spectra of simulated processes have been very different, so a logarithmic PDF was chosen as a compromising solution. However, in the present problem, we have a more favorable situation that allows us to optimize our choice. As it follows from Eq. (10), all the theoretical spectra are the same, so from the viewpoint of simulation accuracy, the best choice is to take $\Omega(\kappa)$ proportional to the square root of $\left(\kappa^{2}+1 / L_{0}^{2}\right)^{-11 / 6}$. Such a distribution can be generated making use of, for example, the rejection method (Macakill \& Beran 1984); however, it is not very convenient from a computational point of view, because it will make the simulation slower. It is therefore preferable to choose a PDF in such a way that the following conditions hold:

(i) a PDF provides a suitable approximation for the square root of $\left(\kappa^{2}+1 / L_{0}^{2}\right)^{-11 / 6}$

(ii) the indefinite integral of this PDF is known analytically and analytically invertible. 
The distribution satisfying the requirements (i) and (ii) is the Lorentzian PDF (Macakill \& Beran 1984), which is given by:

$$
\begin{aligned}
& \Omega(\kappa)=\left\{\begin{array}{cc}
\frac{1}{\Omega_{0}\left(\kappa^{2}+1 / L_{0}^{2}\right)}, & K_{1} \leq \kappa \leq K_{2}, 0 \leq \theta \leq 2 \pi, \\
0, & \text { otherwise }
\end{array}\right\}, \\
& \Omega_{0}=\pi \ln \frac{K_{2}^{2}+1 / L_{0}^{2}}{K_{1}^{2}+1 / L_{0}^{2}},
\end{aligned}
$$

where $\kappa$ and $\theta$ are the modulus and the polar angle of the vector $\kappa$, and $K_{1}$ and $K_{2}$ are the lower and the upper boundaries of the region of spatial frequencies inside which the phase fluctuations are simulated.

In what follows we also need the PDF $\Omega_{\kappa}(\kappa)$ of the modulus $\kappa$ of vector $\boldsymbol{\kappa}$. Because the PDF $\Omega(\boldsymbol{\kappa})$ is an isotropic function of vector $\kappa, \Omega_{\kappa}(\kappa)$ is given by

$\Omega_{\kappa}(\kappa)=\frac{2 \pi \kappa}{\Omega_{0}\left(\kappa^{2}+1 / L_{0}^{2}\right)}$.

With this step, we have calculated all the quantities needed for the simulation. Using the results of these calculations, we can describe a step-by-step simulation receipt as follows.

1. The set $\left\{s_{m}\right\}$ of $M$ random numbers $s_{m}$ distributed uniformly inside the range $[0,1]$ is generated. Then, in order to get the set of $\left\{p_{m}\right\}$ distributed with the PDF (21), the following transformation is applied:

$$
p_{m}=\sqrt{\frac{\left(K_{2}^{2}+1 / L_{0}^{2}\right)^{s_{m}}}{\left(K_{1}^{2}+1 / L_{0}^{2}\right)^{s_{m}-1}}-1 / L_{0}^{2}} .
$$

Randomly generating randomly $M$ angles $\theta_{m}$, uniformly distributed inside the range $[-\pi, \pi]$, we obtain $M$ random vectors $\boldsymbol{p}_{m}$ with PDF given by Eq. (20).

2. Making use of the set $\left\{p_{m}\right\}$ simulated at step 1, we calculate the magnitudes of $W_{S_{l} S_{l}}\left(p_{m}\right)$ and $\Omega\left(p_{m}\right)$ from Eqs. (10) and (20), respectively. It follows from Eq. (10) that the $W_{S_{l} S_{l}}$ do not depend on $l$, so the magnitude of $W_{S_{l} S_{l}}\left(p_{m}\right)$ is calculated only once. Substituting $W_{S_{l} S_{l}}\left(p_{m}\right)$ and $\Omega\left(p_{m}\right)$ into Eq. (17), we get the amplitudes $F\left(p_{m}\right)$.

3. $M$ random magnitudes of $\psi_{m}$ distributed with PDF (19) are generated for any given $C_{n}^{2}$ profile.

4. $M$ random, uniformly distributed inside the range $[-\pi, \pi]$ magnitudes of $\varphi_{m}$ are generated.

5. Substituting the sets of $\left\{F\left(p_{m}\right)\right\},\left\{\boldsymbol{p}_{m}\right\},\left\{\varphi_{m}\right\}$, and $\left\{\psi_{m}\right\}$ into Eq. (12), the samples $S_{l}(\boldsymbol{r})(l=1, \ldots, L)$ can be calculated at any chosen grid of coordinates $\boldsymbol{r}$.

Note that there is no necessity to calculate the magnitudes $W_{S_{l} S_{j}}\left(p_{m}\right)$ of the cross-spectra in the simulation because all the needed cross-correlations are provided by the special choice (19) of the PDF of $\psi_{m}$. This is an additional advantage of the PDF (19) allows the simulation to be accelerated significantly .

In some applications, the simulation of samples of the point spread function to be produced by $L$ stars at the focal plane is of interest. Since the wave fields coming from different stars are incoherent and the amplitude fluctuations are practically negligible under the weak-turbulence conditions [9], these samples $I$ can be calculated as

$$
\begin{aligned}
& I(\boldsymbol{\eta})=\sum_{l=1}^{L} A_{l}^{2} V_{l}(\boldsymbol{\eta}) V_{l}^{*}(\boldsymbol{\eta}), \\
& V_{l}(\boldsymbol{\eta})=\stackrel{\wedge}{\boldsymbol{F}}\left\{\exp \left[i S_{l}(\boldsymbol{r})+i k \boldsymbol{r} \cdot n_{l}\right]\right\},
\end{aligned}
$$

where $\boldsymbol{\eta}$ denotes the 2-D position vector in the focal plane, the asterisk $(*)$ denotes the complex conjugation, $A_{l}$ is the amplitude of $l$ th wave at the upper boundary of the atmosphere, $S_{l}$ the sample of the phase fluctuation of $l$ th star at the aperture, $\hat{\boldsymbol{F}}$ denotes the operator of the two-dimensional Fourier transform, $k$ is the wavenumber, and the expression for $\boldsymbol{n}_{l}$ is presented in Eq. (1).

\section{Simulation results}

To demonstrate the efficiency of the approach developed for the many-stars case, it is sufficient to consider the case of three stars. In this case the cross-correlation functions which have to be reproduced in simulation are: $B_{S_{1} S_{1}}, B_{S_{1} S_{2}}, B_{S_{1} S_{3}}$, and $B_{S_{2} S_{3}}$. We compare the simulated and theoretical functions below for a varying $C_{n}^{2}$ along the propagation path. The simulation parameters have been chosen as follows: the number of harmonics $M=100$, the frequency limits $K_{1}=0$, and $K_{2}=10^{4} 1 / \mathrm{m}$, the number of samples is equal 10000 .

The number of harmonics $M=100$ has been chosen after many sets of preliminary simulations with various simulation parameters. These simulations shows that when the number of harmonics reaches 100, a future increase in this number slightly affects the statistical properties of the simulation results. A more detailed discussion what concern the number of harmonics is presented in Kouznetsov \& Voitsekhovich (1998).

As an example of simulation with a varying $C_{n}^{2}$, we present the simulation results from the Hufnagel $C_{n}^{2}$ profile (Hufnagel 1974):

$$
C_{n}^{2}(z)=C_{0} r_{0}^{-5 / 3} k^{-2}\left[\left(\frac{z}{z_{0}}\right)^{10} \exp \left\{-\frac{z}{z_{1}}\right\}+\exp \left\{-\frac{z}{z_{2}}\right\}\right],
$$

where $r_{0}$ is the Fried parameter, $C_{0}=1.027 \times 10^{-3} \mathrm{~m}^{-1}$, $z_{0}=4.632 \times 10^{3} \mathrm{~m}, z_{1}=10^{3} \mathrm{~m}, z_{2}=1.5 \times 10^{3} \mathrm{~m}$.

In Eq. (28), the altitude $z$ and the Fried parameter $r_{0}$ are given in meters, the wavenumber $k$ is given in inverse meters, and the units of $C_{n}^{2}$ are meters to the power $-2 / 3$.

The PDF $\eta\left(\psi_{m}\right)$ is obtained substituting Eqs. (27) into (19). The $\psi_{m}$ are generated making use of the rejection method (Macakill \& Beran 1984). To apply the rejection method, we use a simplest exponential function as a comparison function $f_{\mathrm{c}}$ :

$f_{\mathrm{c}}\left(\psi_{m}\right)=\frac{1}{A_{0}} \exp \left(-\frac{\psi_{m}}{\psi_{0}}\right)$

$A_{0}=\int_{0}^{\infty} \mathrm{d} z C_{n}^{2}(z), \psi_{0}=1050 \mathrm{~m}$.

The simulation results are then compared to the theoretical ones in Fig. 1. One can see a good agreement between the 

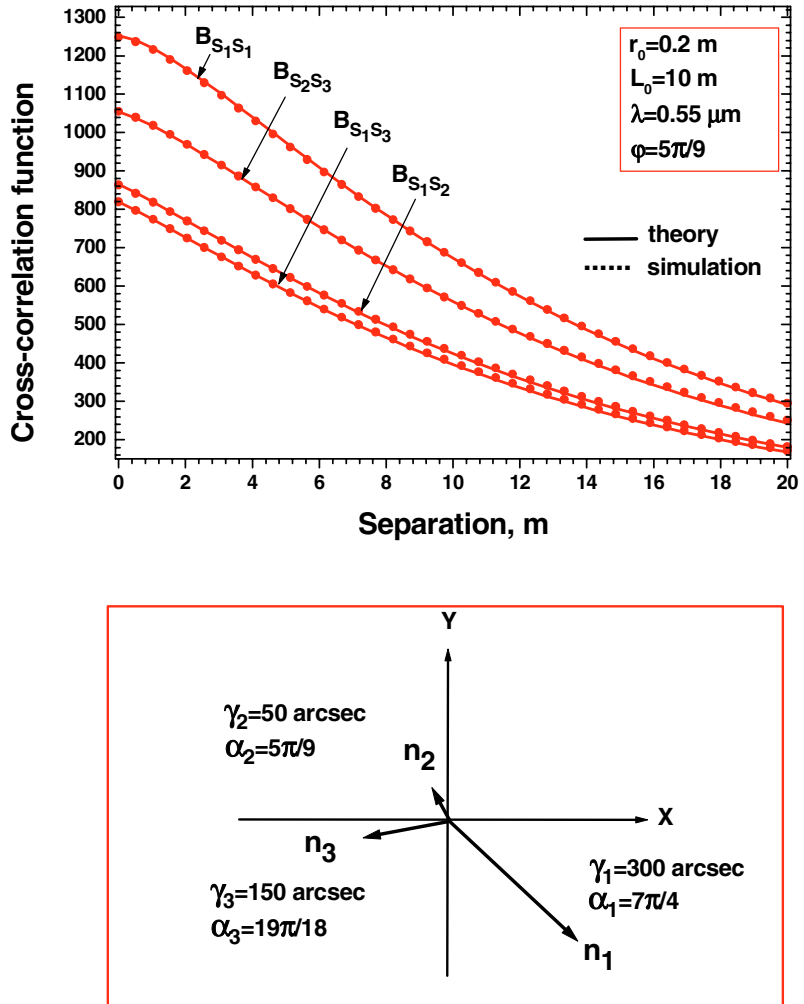

Fig. 1. Comparison of the simulated and theoretical results for the case of three stars (varying $C_{n}^{2}$, the Hufnagel model). The correlation function $B_{S_{1} S_{1}}$, and the cross-correlation functions $B_{S_{1} S_{2}}, B_{S_{1} S_{3}}$ and $B_{S_{2} S_{3}}$ are plotted in the top graph. The cross-correlation functions are anisotropic but homogeneous; i.e. they can be presented as functions of two scalar arguments: the separation $\rho=\left|\boldsymbol{r}_{1}-\boldsymbol{r}_{2}\right|$ between two observation points $\boldsymbol{r}_{1}$ and $\boldsymbol{r}_{2}$, and the polar angle $\varphi$ of the vector $\boldsymbol{r}_{1}-\boldsymbol{r}_{2}$. The angle $\varphi$ is chosen as a parameter in the top graph. The bottom graph shows the locations of the stars thematically. The $\boldsymbol{n}_{l}$ is the projection of $l$ th propagation vector on the aperture plane; $\gamma_{l}$ is the angular separation between $l$ th star and $Z$-axis; $\alpha_{l}$ is the polar angle of the vector $\boldsymbol{n}_{l}$.

theory and simulation; numerically the rms deviation between simulated and theoretical results does not exceed a few percent.

The maximum separation between the observation points at the aperture is chosen equal to $20 \mathrm{~m}$, in order to show where the correlations are dropped strongly. However in simulations related to a real telescope, the samples can be generated at any finite aperture choosing a proper maximum value of in Eq. (12).

\section{Conclusions}

A method suitable for simulating the phase cross-correlated fluctuations produced by many stars has been proposed. It turned out that for the case of the weak-turbulence conditions important for astronomical applications, it is possible to develop a relatively simple and fast procedure simulations. The validity of theoretical derivations has been verified in the simulation with varying $C_{n}^{2}$ along the propagation path. Comparison of theoretical and simulated results has shown that the approach developed reproduces all the needed cross-correlations in simulated samples.
The simulation results have also shown a good coincidence among the theoretical and simulated results for a wide range of separations between the observation points. This means that the method allows reproduction of a wide range of spatial frequencies in simulated processes using a relatively small number of harmonics; only 100 harmonics in the present simulation. The notable property of the method to reproduce a wide range of spatial frequencies is its main advantage over conventional simulation approaches. Let us analyze a physical reason for this advantage of this method over the Fourier approach, which is frequently used in atmospheric simulations (Sedmak 2004; Louthain \& Welsh 1998). Similar to the Fourier technique, the RWV approach suggests constructing the simulated process through its expansion in the domain of spatial frequencies. However, in the Fourier method, the limits of spatial frequencies within which the simulation can be performed are always restricted by the spatial grid. Briefly, the wider is a desired frequency range, the bigger the grid has to be in the simulation. In contrast, the RWV technique use a more flexible approach, and the main difference is in the choice of discrete frequencies. These frequencies (random wave vectors) are not related to the spatial grid at all. Instead they are generated randomly with a distribution matching the spectrum of simulated process. A range of spatial frequencies to be reproduced in simulation is determined by the limits within which the random wave vectors are generated. Since these limits can be chosen arbitrary, it allows us to simulate the processes with very wide spectra. For example, in the present simulation these limits have been chosen from 0 to $10^{4} 1 / \mathrm{m}$.

Generalizing from these considerations, we can conclude that the main advantage of this approach is in the probale choice of spatial frequencies. In some sense, the method works in a similar way to the real atmosphere which produces the distortions that "assign" a given probability that each spatial frequency will occur. And the close a distribution of simulated random wave vectors is to the natural one, the better natural statistics are reproduced in simulated samples. Actually, this similarity is the main physical reason the makes RWV approach is so successful in simulating random processes.

The good agreement between the simulated and theoretical results, even for big separations allows, a wide range of possible applications of this method. Among them are adaptive optics, guide stars, long-base interferometry, speckle interferometry, imaging through the turbulence, as well as simulations of the turbulence-degraded images of extended objects.

Acknowledgements. This work was supported by Direccion General de Asuntos del Personal Academico (UNAM, Mexico) under project IN126802-2 (PAPIIT).

\section{References}

Berkefeld, T., Glindemann, A., \& Hippler, S. 2001, Exper. Astron., 11,1

Cannon, R. C. 1995, J. Opt. Soc. Am. A, 13, 862

Dainty, J. C. 1974, MNRAS, 169, 631

Foy, R., \& Laberye, A. 1985, A\&A, 152, L29

Fried, D. L. 1982, J. Opt. Soc. Am., 72, 52

Fried, D. L. 1995, J. Opt. Soc. Am. A, 12, 939 
Fusco, T., Conan, J-M., Michau, V., et al. 2001, Proc. SPIE, 4167, 168

Hufnagel, R. E. 1974, Optical Propagation through Turbulence, OSA Technical Digest Series (Washington, DC: OSA), WA1-1

Korff, D. 1973, J. Opt. Soc. A., 63, 1971

Kouznetsov, D., Voitsekhovich, V. V., \& Ortega-Martinez, R. 1997, Appl. Opt., 36, 464

Kouznetsov, D., \& Voitsekhovich, V. V. 1998, Meteorol. Z. N.F., 7, 230

Louthain, J. A., \& Welsh, B. M. 1998, Proc. SPIE, 3381, 286

Macakill, C., \& Beran, M. J. 1984, IMA J. Appl. Math., 33, 1

Martin, J. M., \& Flatte, S. M. 1988, Appl. Opt., 27, 2111

Orlov, V. G., Voitsekhovich, V. V., \& Cuevas, S. 1998, Appl. Opt., 37, 4544

Press, W. H., Flannery, B. P., Teukolsky, S. A., et al. 1995, Numerical Recipes in C, Second edition, Ch. 7, (Cambridge: Cambridge University Press)
Reinhardt, G. W., \& Collins, S. A. 1972, J. Opt. Soc. Am., 62, 1526

Ricklin, J. C., Vorontsov, M. A., Carhart, G. W., et al. 1995, J. Mod. Opt., 42, 13

Roddier, F. 1981, Progr. Opt., 19, 281

Roddier, F., Gilli, J. M., \& Vernin, J. 1982, J. Opt., Paris, 13, 63

Roddier, N. 1990, Opt. Eng., 29, 1174

Schock, M., Foy, R., Tallon, M., et al. 2002, MNRAS, 337, 910

Sedmak, G. 2004, Appl. Opt., 43, 4527

Shapiro, J. H. 1976, J. Opt. Soc. Am., 66, 460

Tallon, M., \& Foy, R. 1990, A\&A, 235, 549

Tatarski, V. I. 1961, Wave Propagation in a Turbulent Medium (New York: McGraw-Hill)

Voitsekhovich, V. V., Orlov, V. G., Cuevas, S., \& Avila, R. 1998, A\&AS, 133, 427

Vorontsov, M. A., Carhart, G. W., Pruidze, D. V., et al. 1996, J. Opt. Soc. Am. A, 13, 1456 